\title{
Lifetime Improvement of Dynamic Under Water Acoustic Sensor Network
}

\author{
R. Sowbarani, M. Sivakumar
}

Adhiyamaan College of Engineering, Dr. M.G.R. Nagar, Hosur- 635109

\begin{abstract}
In underwater acoustic sensor network [UASN], sensors life time increment is the main problem, manually we cannot recharge the battery by going deep into the sea or ocean and we cannot recharge it by solar power since sun light won't go deep into the sea. In previous work on UASN they considers only static network, but in this paper we consider dynamic network (changes by time, either the sensor is active, malfunctioned, dead or lost) and in previous work they took $2 D$ model and in this we consider a $3 D$ model for sensing the target. To improve the life time of Underwater Acoustic Sensor Network (UASN) we developed a Heuristic Search Algorithm (Multi-population Harmony Search Algorithm) to dynamically choose to sleep or work a given set of sensors in order to cover the given set of targets.
\end{abstract}

Keywords: Underwater acoustic sensor network, harmony search algorithm, multi-population, dynamic optimization, pitch adjusting rate

\section{Introduction}

In underwater acoustic sensor network sensors are placed underwater to make a wireless network frame to discover new resources, detect targets and monitor pollution.

In general UASN heterogeneous wireless sensor, sensor nodes and acoustic waves to transmit and autonomous underwater vehicles (AUVs) with pumps are present.

From Fig: 1 the transmission process is known clearly. Initially from the bottom sensor (which is placed at the bed of the ocean), the targets are detected and the signal is passed to the AUV and then to underwater sensor. From that to the surface sink (which is placed at the ocean surface). And then to the base station which is placed at the earth surface.

In this method sensors are classified into multiple disjoint covers, each of which is a subset of sensors to cover all targets. If more sensor covers are there mean lifetime of the WSN can be increased, since each sensor cover can have a backup for an inactive cover.

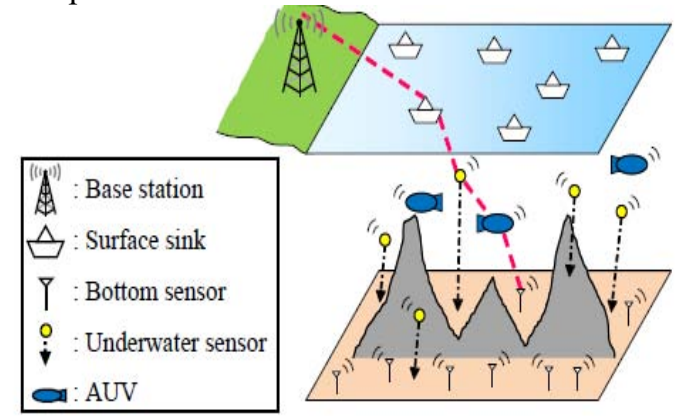

Fig: 1 Illustration of underwater acoustic sensor networks.

\section{UASN}

Acoustic communication is used to transmit signals in underwater. In fact, radio wave will transmit more than acoustic but in sea water it requires big antennas and high transmitting rate. Optical waves do not support such high transmission rate.
A. Challenges of UASN

- Limited bandwidth.

- Impaired channel.

- High Propagation delay.

- Bit error rate is high.

- Limited battery power.

- Failure of sensors due to fouling and corrosion.

\section{B. Applications of UASN}

Ocean sampling networks, undersea explorations, Mine reconnaissance, distributed tactical surveillance, Environmental monitoring, Disaster prevention, assisted navigation.

\section{Contribution of the Present Work}

First, this work considers a dynamic problem. Second, positions of some sensors are not fixed the proposed algorithm can dynamically apply the updated positions to make a new sleep schedule.

\section{Basic Harmony Search Algorithm}

Musicians play many harmonies, for various combination of music. Harmony search has two different functions they are Harmony Memory considering Rate (HMCR) and Pitch Adjusting Rate (PAR).

Rules for better harmony in music:

1. Selecting any pitch from memory.

2. Selecting adjacent pitch.

3. Selecting any random pitch.

Similar rules for sensor targeting:

1. Selecting any value.

2. Selecting adjacent value.

3. Selecting any random value.

In Fig: 2 it shows the block diagram of harmony search algorithm and how it works based upon the above rules. 


\section{International Journal of Science and Research (IJSR)}

ISSN (Online): 2319-7064

Index Copernicus Value (2013): 6.14 | Impact Factor (2014): 5.611

The Proposed Method MPHSA

When compared to genetic algorithm, MPHSA is more advanced. HSA is used mainly for dynamic model.

\section{3D Model}

Fig: 3 shows the three dimensional representation of underwater sensor networks. In 3D underwater networks, sensors are allowed to float in water. The sensors are tied with a wire so that the height can be adjusted according to the target.

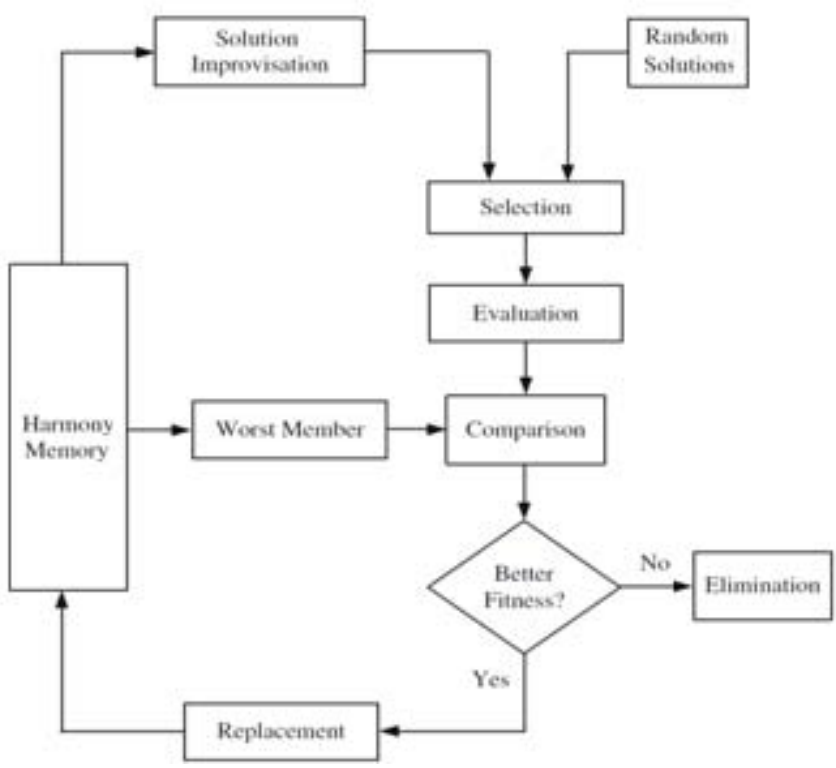

Figure 2: Harmony Search Algorithm

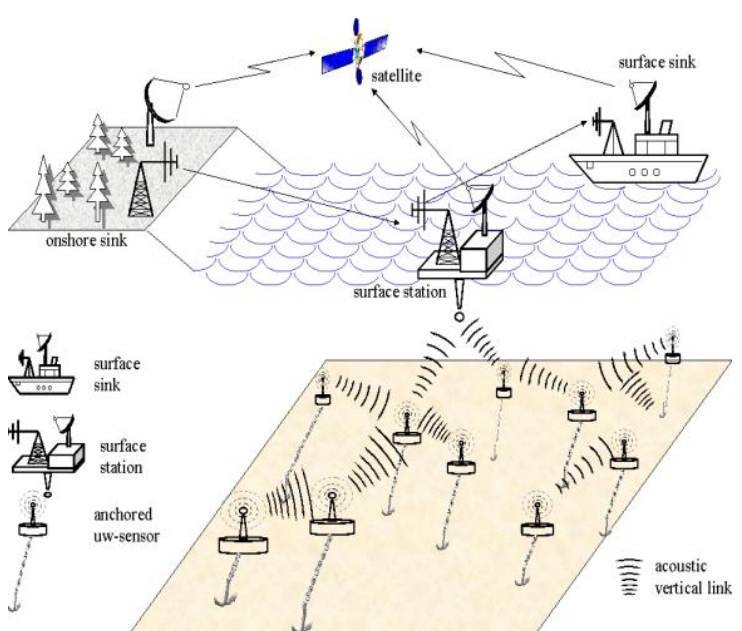

Figure 3: Three-Dimensional representation of UASN

\section{Mathematical Model}

Consider in Fig. 4(a), there are 6 number of sensors $\left(s_{1}-s_{6}\right)$ and 4 number of target $\left(\pi_{1}-\pi_{4}\right)$. Each sensor is arranged in a sphere structure to cover all targets easily. The sensing range size of each sensor may differ due to its heterogeneous sensor type. Base station (BS) is placed, up above the sea level to collect the messages which is transmitted from the sea bed.

At a particular time, each sensor could be in one of four modes: active, asleep, malfunctioned, and dead. Only active sensors will work to detect the targets and consume battery power.To save the battery power, sensors that are not active can be turned off. Sensor may be dead due to battery power depletion, or get lost due to external factors.

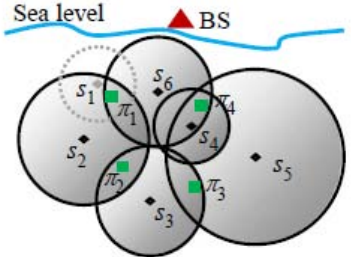

(a) At the $\tau$-th key time

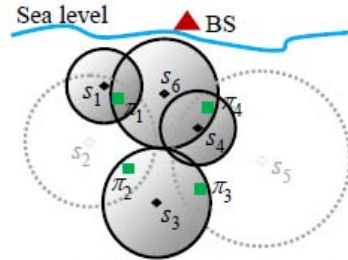

(b) At the $(\tau+1)$-th key time
Figure 4: Example for dynamic UASN at two key times.

Sensors that are active or asleep are called as surviving sensors and sensors that are malfunctioned or deadlines are called to fail.

Sensor modes vary, based upon the active sensors vary at each and every time. So, in this work we propose a method to decide a sleep schedule at each and every key time.

As shown in Fig. 5, the 1st key time is the initial time, at which each sensor is works with the initial battery power. Here the sleep schedule is initialised. During the $1^{\text {st }}$ key if some targets are not covered mean the $2^{\text {nd }}$ key time is started.

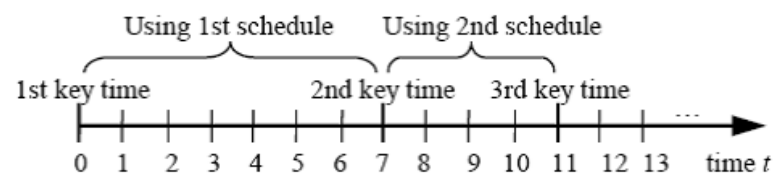

Figure 5: Relationship between sleep schedule and key time.

At the 2nd key time, the sensors information is updated and sleep schedule is followed to cover all targets.

Similarly, the 3rd key time, 4th key time and so on can be followed. And, a sleep schedule is followed at each key time until survival sensors cannot cover all targets.

\section{Notations Used}

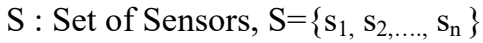

$\mathrm{T}:$ Set of Targets, $\mathrm{T}=\{\pi 1, \pi 2, \ldots \mathrm{n}\}$

$\mathrm{S}_{\tau}$ : Set of survival sensors at the $\tau$-th key time

$\mathrm{F}_{\tau}$ : Set of malfunctioned sensors at the $\tau$-th key time

$\mathrm{D}_{\tau}$ : Set of dead sensors at the $\tau$-th key time.

$\mathrm{R}_{\tau}$ : Set of sensors that are recovered at the $\tau$-th key time.

$\mathrm{P}_{\mathrm{f}}$ : Malfunction probability

$\mathrm{P}_{\mathrm{d}}$ : Dead probability

$\mathrm{P}_{\mathrm{r}}$ : Recovery probability

$\mathrm{S}=\left\{\mathrm{s}_{1}, \mathrm{~s}_{2}, \ldots, \mathrm{s}_{\mathrm{n}}\right\}$ in a UASN to detect all targets in $\mathrm{T}=\left\{\pi_{1}\right.$, $\left.\pi_{2}, \ldots, \pi_{\mathrm{m}}\right\}$ at different key times. Consider the sleep schedule at the $\tau$-th key time. Initially update the set of survival sensors $(\mathrm{S} \tau)$ at $\tau$-th key time.

$$
\mathrm{S} \tau=(\mathrm{S} \tau-1 \mathrm{U} \mathrm{R} \tau) \backslash(\mathrm{F} \tau \cap \mathrm{D} \tau)
$$

\section{MPHSA Algorithm}

1. At the $\tau$-th key time survival sensors are updated 


\section{International Journal of Science and Research (IJSR) \\ ISSN (Online): 2319-7064}

Index Copernicus Value (2013): 6.14 | Impact Factor (2014): 5.611

2. Initialize the parent harmony memory HM.

3. Divide HM into sub-HM (sub-HM1, sub-HM2, ..., sub-HM $\delta$ )

4. Initialize the current iteration number as 1( ie., $\eta=1,2, \ldots, n)$

5. if rand $(0,1)<$ HMCR then

6. Choose two harmonies $\mathrm{x}^{\text {new1 }}$ and $\mathrm{x}^{\text {new2 }}$ from sub-HMi

7. if $\operatorname{rand}(0,1)<\operatorname{PAR}(\eta)$ then

8. Make a uniform crossover operator on $x^{\text {new1 }}$ and $x^{\text {new2 }}$, and replace the resultant value in te place of $x^{\text {new1 }}$ and $\mathrm{x}^{\text {new2 }}$.

9. end if

10. Let $x^{\text {new }}$ be the one of $x^{\text {new1 }}$ and $x^{\text {new2 }}$ with a better fitness value

11. else

12. Randomly generate a feasible harmony as $\mathrm{x}^{\text {new }}$

13. end if

14. If $x^{\text {new }}$ is better than worst harmony in sub-HMi, $x^{\text {new }}$ replaces it

15. end for

16. $\eta=\eta+1$

17. end while

18. Decode the best harmony among all sub-HMi's

19. If number of covers is non zero, randomly choose one of the covers as the output, otherwise output is zero i.e., no solution.

\section{Simulation Result}

By using different number of sensors and targets we can run the MPHSA algorithm to get various outputs. And the number of iterations can also be extended until we get better solution to detect the target.

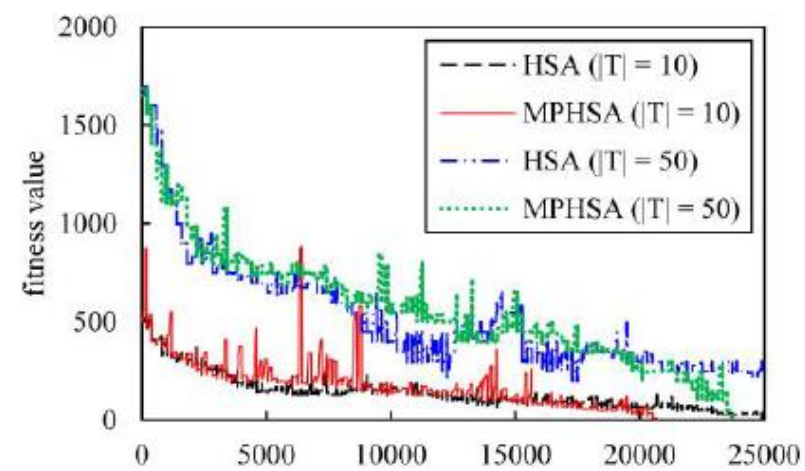

Figure 6: Experimental comparisons when number of sensors $|S|$ is 100 or 500 .

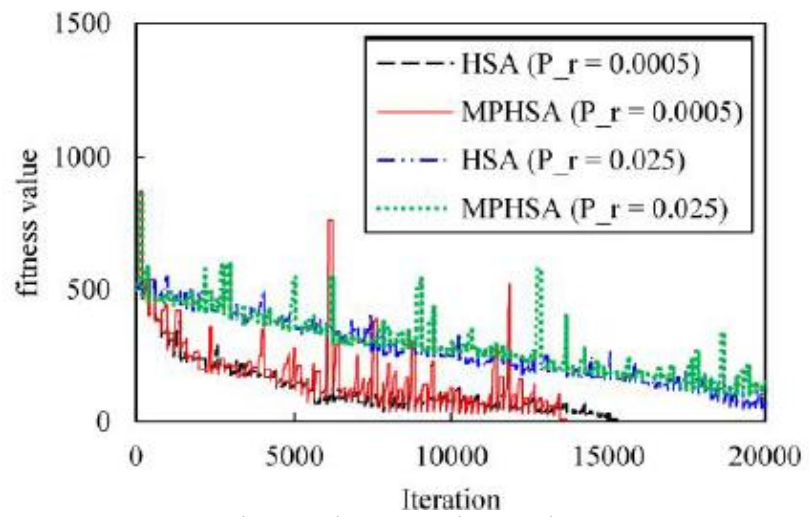

Figure 7: Experimental comparison when $P f=0.0005$ or 0.025 .

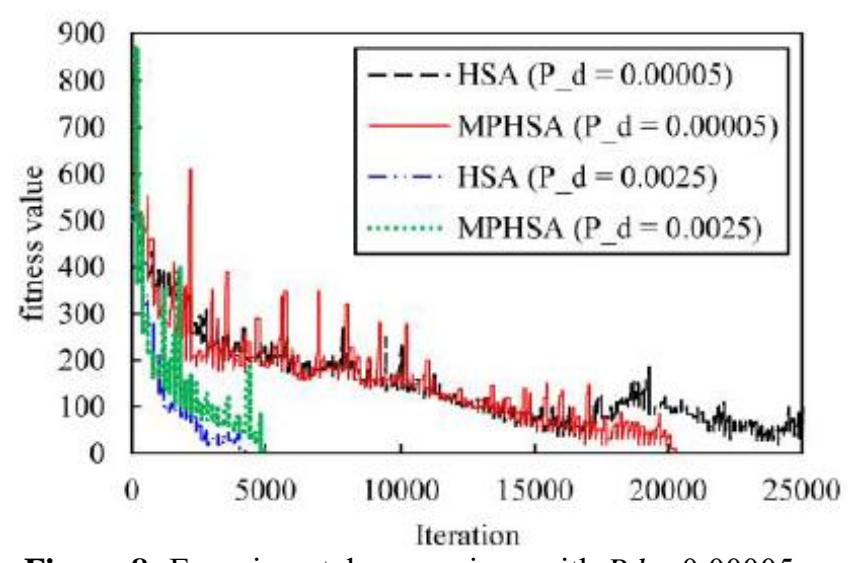

Figure 8: Experimental comparison with $P d=0.00005$ or 0.0025 .

\section{Conclusion}

In this work we dynamically determined a sufficient number of active nodes in the UASN at different times to develop a sleep scheduling scheme. This work develops a MPHSA which yields better results than Genetic Algorithm. This works considers 3D Architecture of UASNs for more coverage area.

\section{References}

[1] Chun-Cheng Lin, Member, IEEE, Der-Jiunn Deng, Member, IEEE, and Shang-Bin Wang "Extending the life time of Dynamic Underwater Acoustic Sensor Networks using Multi-population Harmony Search Algorithm" 10.1109/JSEN.2015.2440416, IEEE Sensors Journal

[2] P. Salvo Rossi, D. Ciuonzo, T. Ekman, and H. Dong, "Energy detection for MIMO decision fusion in underwater sensor networks," IEEE Sensors J., vol. 15, no. 3, pp. 1630-1640, 2015.

[3] M. Castelli, S. Silva, L. Manzoni, and L. Vanneschi, "Geometric selective harmony search," Inform. Sciences, vol. 279, pp. 468-482, 2014.

[4] S. Iyer and D. V. Rao, "Genetic algorithm based optimization technique for underwater sensor network positioning and deployment," in Proc. IEEE UT, 2015, pp. 1-6.

[5] W. Jiang, J. Wang, W. Wang, L.-L. Cao, and Q. Jin, “A parallel harmony search algorithm with dynamic harmony-memory size," in Proc. CCDC, IEEE Press, 2013, pp. 2342-2347.

[6] S. Ibrahim, J. Liu, M. Al-Bzoor, J.-H. Cui, and R. Ammar, "Towards efficient dynamic surface gateway deployment for underwater network," Ad Hoc Netw., vol. 11, pp. 2301-2312, 2013.

[7] G. Brataas, A. Lie, and T.A. Reinen, "Scalability analysis of underwater sensor networks," in Proc. MTS / IEEE. Conf. Oceans, 2013, pp. 1-9. 